\title{
Reaching desired states time-optimally from equilibrium and vice versa for visco-elastic joint robots with limited elastic deflection
}

\author{
Nico Mansfeld and Sami Haddadin
}

\begin{abstract}
Recently, intrinsically elastic joints became increasingly popular due to several reasons. Most importantly, elasticity improves impact robustness and, if used wisely, energy efficiency. Potential energy storage and release capabilities in the joints allow to outperform rigid manipulators by means of achievable peak link velocity. It has therefore been of great interest to find explosive or cyclic motions, similar to those of humans or animals, that make systematic use of joint elasticity. In this context, we address two important control problems in the present paper. First, we find all potential system states that a visco-elastic joint with constrained deflection may reach from its equilibrium state and analyze the influence of system parameters on the according reachable set. While high link velocities are certainly desirable in terms of performance, they may also increase the robot's level of dangerousness and/or the risk of self damage during potentially unforeseen collisions. Thus, we tackle the problem of how to brake a visco-elastic joint in minimum time. Furthermore, the results are extended to a near-optimal real-time control law for elastic $n$-DOF manipulators. The proposed braking controller is experimentally verified on a KUKA/DLR LWR4 in joint impedance control.
\end{abstract}

\section{INTRODUCTION}

Many intrinsically compliant robotic mechanisms were proposed over the past few years [1], [2], [3], [4], [5]. These designs aim at improving robustness, safety in humanrobot interaction, and energy storage for highly dynamic and/or energy efficient motions. The introduction of elasticity opens completely new and often unforeseen control problems in robotics. For outperforming classic rigid actuation by means of reachable peak velocity, optimal control theory has been addressed recently. Generating optimal trajectories for full robotics systems such as the DLR Hand Arm System typically requires numerical methods due to high complexity [6]. Analytic solutions have therefore mainly been found for the 1-DOF case.

First insights for accelerating undamped elastic joints optimally were given in [7], [8], the influence of damping was afterwards analyzed in [9]. While these works considered unconstrained systems, [10] derived the maximum possible velocity for an elastic joint that has limited elastic deflection, i.e. storable energy. In this line of research, we extend our previous work. Instead of aiming at peak velocities only, we consider the more general problem of which states we can reach from equilibrium with an elastic joint while taking limited elastic deflection, motor velocity, and damping into account.

While high velocities are desirable for performance improvement, they also increase the potential threat of a robot

Nico Mansfeld is with the Institute of Robotics and Mechatronics, German Aerospace Center (DLR), Wessling, Germany, Sami Haddadin is with the Institute of Automatic Control, Leibniz University Hannover (LUH), Germany, nico.mansfeldedlr.de, sami .haddadin@irt.uni-hannover.de

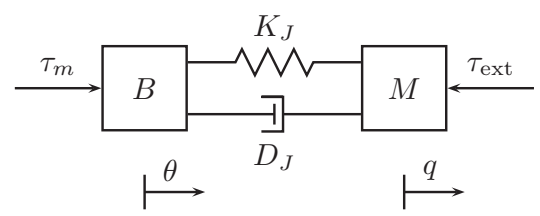

Fig. 1. 1-DOF visco-elastic joint

in case of a collision [11]. The second problem we address in this paper is therefore how to stop an elastic robot as fast as possible. We find solutions for the 1-DOF case and extend the problem to braking of $n$-DOF manipulators. We propose a controller for braking in near-minimum time and verify its performance experimentally.

\section{PROBLEM FORMULATION}

\section{A. Considered model}

Usually, a visco-elastic joint is modeled as follows, see Fig. 1. It consists of a motor with inertia $B$ and associated position $\theta$, constant joint damping $D_{J}$ and joint stiffness $K_{J}$, and a link with position $q$ and inertia denoted by $M$. The overall dynamics can be expressed in terms of following differential equations

$$
\begin{aligned}
B \ddot{\theta} & =\tau_{m}-\tau_{f}-\tau_{J} \\
M \ddot{q} & =\tau_{g}+\tau_{\mathrm{ext}}-\tau_{J} \\
\tau_{J} & =K_{J}(\theta-q)+D_{J}(\dot{\theta}-\dot{q}),
\end{aligned}
$$

where $\tau_{m}, \tau_{f}, \tau_{g}, \tau_{\text {ext }} \in \mathbb{R}$ are the motor, motor friction, link gravity, and external torques. For sake of simplicity and clarity of the analysis, we assume $\tau_{f} \approx 0$ and $\tau_{\text {ext }} \approx 0$. Also, we consider the joint to move horizontally, i.e. $\tau_{g}=0$. We take following constraints on the spring deflection and motor velocity into account:

$$
\begin{aligned}
|\varphi| & =|\theta-q| \leq \varphi_{\max } \\
|\dot{\theta}| & \leq \dot{\theta}_{\max }
\end{aligned}
$$

Generally, it is assumed that the motor can apply the maximum velocity (5) under all possible operating conditions.

For intrinsically elastic joints, the motor dynamics are typically much faster than the link side dynamics. If stiff velocity control is chosen, one can bring the dynamics into singular perturbation form and simplify them by modeling the motors as velocity sources [6]. Of course, one should take the maximum motor torque into the consideration as well. However, this would complicate the analysis substantially. Therefore, we only consider the velocity constraint, as it is the more important one. The reduced dynamics are found to 
be

$$
\begin{aligned}
& \theta=\int \dot{\theta} d t+\theta_{0} \\
& \ddot{q}=2 D \omega(\dot{\theta}-\dot{q})+\omega^{2}(\theta-q),
\end{aligned}
$$

with $\theta_{0}$ being the initial motor position, $\omega=\sqrt{K_{J} / M}$ the undamped eigenfrequency, and $D=D_{J} /\left(2 \sqrt{K_{J} M}\right)$ the damping ratio. Damping is typically undesired in elastic joints designed for performance increase and kept as low as possible, because it deteriorates velocities. Therefore, we only consider underdamped joints $(D<1)$ in this work.

For describing the first order differential equations we select the system state to be $x:=\left[\begin{array}{ll}\dot{q} & \varphi\end{array}\right]^{T}$. Now (6) and (7) can be grouped as

$$
\dot{\boldsymbol{x}}=A \boldsymbol{x}+B u=\left(\begin{array}{cc}
-2 D \omega & \omega^{2} \\
-1 & 0
\end{array}\right) \boldsymbol{x}+\left(\begin{array}{c}
2 D \omega \\
1
\end{array}\right) u,
$$

where $u:=\dot{\theta}$ is the control input.

Next, we formally define both control problems considered in this paper, which are reaching desired states from equilibrium (problem I) and vice versa (problem II).

\section{B. Problem I}

The reachable set $\mathcal{R}$ is defined as the set of states, that can be reached from the origin, i.e. $\boldsymbol{x}(0)=\left[\begin{array}{ll}0 & 0\end{array}\right]^{T}$ without violating any constraint (4), (5) and without external forces being applied. Together with the initial and terminal conditions the first problem is fully determined as

$$
\begin{aligned}
& \dot{q}(0)=0, \quad \dot{q}\left(t_{f}\right)=\dot{q}_{d} \\
& \varphi(0)=0, \quad \varphi\left(t_{f}\right)=\varphi_{d},
\end{aligned}
$$

where $\dot{q}_{d}$ and $\varphi_{d}$ are the desired link velocity and deflection at final time $t_{f}$. The desired state is assumed to be located within the reachable set, i.e. $\boldsymbol{x}_{d}:=\left[\dot{q}_{d} \varphi_{d}\right]^{T} \in \mathcal{R}$.

\section{Problem II}

The set of brakable states $\mathcal{B}$ is defined as the set that contains states which can be brought to zero link velocity and deflection without violating the path constraints (4) and (5). A brakable state can either be one that was reached by a trajectory from problem I or due to some external contact, i.e. $\tau_{\text {ext }} \neq 0$. Potentially, these states that cannot be reached via system dynamics alone. The initial and final conditions for this problem are

$$
\begin{array}{cc}
\dot{q}(0)=\dot{q}_{0}, & \dot{q}\left(t_{f}\right)=0 \\
\varphi(0)=\varphi_{0}, & \varphi\left(t_{f}\right)=0,
\end{array}
$$

where $\boldsymbol{x}_{0}:=\left[\begin{array}{ll}\dot{q}_{0} & \varphi_{0}\end{array}\right]^{T} \in \mathcal{B}$ is the initial state.

Given feasible initial and final conditions for both problems, we seek for the time-optimal trajectories to fulfill them, while meeting the system constraints. For this, the according minimum-time optimal control problems are formulated next.

\section{Optimal control formulation}

Intuitively speaking, we seek to accelerate or slow down the joint as fast as possible. The cost function in both cases is simply $\min J(u)=t_{f}$, subject to the dynamics (8). The control input is the limited motor velocity $u:=\dot{\theta},|u| \leq$ $u_{\max }=\dot{\theta}_{\max }$. The Hamiltonian is then defined as

$$
H=\left(2 D \omega\left(u-x_{1}\right)+\omega^{2} x_{2}\right) \lambda_{1}+\left(u-x_{1}\right) \lambda_{2},
$$

where $\lambda_{1}$ and $\lambda_{2}$ are the costates. In addition to the bounded input, also a state constraint has to be considered, namely the limited elastic deflection (4). Formally, one constraint represents the upper and one the lower maximum deflection, respectively

$$
\boldsymbol{h}=\left(\begin{array}{l}
h_{1} \\
h_{2}
\end{array}\right)=\left(\begin{array}{c}
\theta-q-\varphi_{\max } \\
-\theta+q-\varphi_{\max }
\end{array}\right) \leq\left(\begin{array}{l}
0 \\
0
\end{array}\right) .
$$

The state inequality constraint can e.g. be taken into account by directly adjoining it to the Hamiltonian (14) to form an extended Hamiltonian ${ }^{1}$. In this paper, we omit the full optimal control formalism for sake of brevity and readability. We focus on the formed plots that are really necessary to understand the given line of argumentation. As will be shown later, the considered problems can be divided into subproblems using some physical reasoning. The solutions for these subproblems can then be found by exploiting the solution without state constraint and the necessary boundary control in case of active state constraint.

1) Unconstrained problem: If no state constraint is active, the costate dynamics are $\dot{\lambda}_{1}=2 D \omega \lambda_{1}+\lambda_{2}$, and $\dot{\lambda}_{2}=$ $-\omega^{2} \lambda_{1}$. Rewriting the dynamics yields $\ddot{\lambda}_{1}-2 D \omega \dot{\lambda}_{1}+\omega^{2} \lambda_{1}=$ 0 . The resulting switching function is

$$
\sigma=\frac{\partial H}{\partial u}=2 D \omega \lambda_{1}+\lambda_{2}=\dot{\lambda}_{1} .
$$

As the control input enters the Hamiltonian linearly, the optimal control law according to the minimum principle of Pontryagin becomes:

$$
u^{*}= \begin{cases}-u_{\max } \operatorname{sign}\left(\dot{\lambda}_{1}\right), & \dot{\lambda}_{1} \neq 0, \\ \operatorname{singular}, & \dot{\lambda}_{1}=0\end{cases}
$$

Singular solution arcs can be excluded, due to the controllability matrix $C=\left[\begin{array}{ll}B & A B\end{array}\right]$ having full rank [13]. Thus, $u^{*}$ is of bang-bang type. Solving the costate dynamics and inserting them into (15) yields

$$
\sigma=e^{D \omega t}\left(c_{1} \cos \left(\omega_{d} t\right)+c_{2} \cos \left(\omega_{d} t\right)\right),
$$

where $\omega_{d}=\sqrt{1-D^{2}} \omega$ is the damped eigenfrequency, $c_{1}$ and $c_{2}$ are constants depending on the initial costate values, damping ratio and eigenfrequency. Since the switching function is $2 \pi$-periodic, a zero crossing occurs the latest every half period $\pi$. In direct consequence, the switching time of the bang-bang controller is $t_{s}=\pi / \omega_{d}$.

2) Boundary control: Since (14) is differentiated once with respect to time until the input appears explicitly, the constraint is of order 1 .

$$
\frac{d \boldsymbol{h}}{d t}=\frac{d}{d t}\left(\begin{array}{l}
h_{1} \\
h_{2}
\end{array}\right)=\left(\begin{array}{c}
u-x_{1} \\
-u+x_{1}
\end{array}\right) \leq\left(\begin{array}{l}
0 \\
0
\end{array}\right)
$$

If the constraint is active, (18) yields that the boundary control becomes $u_{b}=x_{1}$. When the motor and link travel at the same velocity, the relative position $x_{2}=\theta-q$ remains

\footnotetext{
${ }^{1} \mathrm{~A}$ comprehensive survey on dealing with state constraints can be found in [12].
} 
constant. After hitting $x_{2}=\varphi_{\max }$ this control ensures that the constraint will not be violated. Of course, the motor velocity constraint (5) must be met at any time. Boundary control is therefore only possible if $\left|x_{1}\right| \leq u_{\max }$.

\section{TIME-OPTIMAL CONTROL FOR 1-DOF VISCO-ELASTIC JOINTS}

\section{A. Reaching desired states from equilibrium}

For the first problem of reaching a desired state starting from equilibrium, we can identify three principal cases ( $\mathrm{r} 1$, r2, and r3). Each case has a different set of reachable states. The reachable set is either bounded by

r1) an enclosing limit cycle: all reachable states can be hit by bang-bang control,

r2) the maximum elastic deflection: all reachable states can be hit by bang-bang control, or

r3) the maximum elastic deflection: quasi-singular solution areas may occur.

In the following, all three cases are described and summarized.

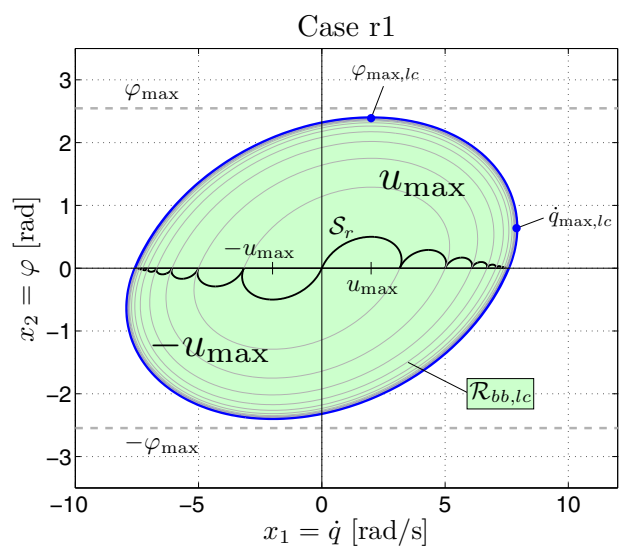

Fig. 2. Reachable states in case the elastic deflection is larger than the maximum deflection of the limit cycle (case r1). The switching manifold is denoted $\mathcal{S}_{r}$. The blue line represents the limit cycle, the colored area all reachable states.

1) Case rl: If damping is present and the elastic deflection unlimited, [9] showed that the system approaches a limit cycle when bang-bang control is applied, see Fig. 2. The switching manifold for time-optimal control, referred to as $\mathcal{S}_{r}$, can be obtained when starting at $\left[\begin{array}{ll}0 & 0\end{array}\right]^{T}$ and applying $u= \pm u_{\max }$ for $t=t_{s}$. Starting a new spiral from every point of these curves defines the adjacent curves on the manifold. Successively repeating this procedure gives the remaining spirals of $\mathcal{S}_{r}$, see Fig. 2 [13]. Above the switching manifold, one must apply $u=u_{\max }$, below $\mathcal{S}_{r} u=-u_{\max }$ applies to hit the steady state in minimum time. From the switching curves it can be observed that the velocity increase reduces for each successive motor cycle due to damping. The maximum possible velocity is

$$
\dot{q}_{\max , l c}=u_{\max }\left(1+2 \frac{F_{1}(D)}{1-F_{2}(D)}\right), \quad \forall D>0,
$$

where

$$
\begin{aligned}
& F_{1}(D)=e^{-\frac{D}{\sqrt{1-D^{2}}}\left(\pi-\operatorname{atan} 2\left(2 D \sqrt{1-D^{2}}, 1-2 D^{2}\right)\right)}, \\
& F_{2}(D)=e^{-\frac{\pi D}{\sqrt{1-D^{2}}}} .
\end{aligned}
$$

This velocity can only be obtained by an infinite number of motor cycles. The reachable states, denoted $\mathcal{R}_{b b, l c}$, are all states that are enclosed by the limit cycle (colored area in Fig. 2). Because the deflection constraint is inactive for all $\boldsymbol{x}_{d} \in \mathcal{R}_{b b, l c}$, all states can be reached by the bang-bang control.

The maximum velocity increase $\epsilon=\dot{q}_{\max } / u_{\max }$ for this case as well as for $\mathrm{r} 2$ and $\mathrm{r} 3$ is depicted in Fig. 3. It depends on the damping ratio $D$ and $\sqrt{e_{S L}}=\frac{\omega \varphi_{\max }}{\dot{\theta}_{\max }}$, a measure for the energetic capability of the joint which is defined in [10].

For large $\sqrt{e_{S L}}$, the kinetic energy provided by the motor is low in comparison to the potential spring energy. In this case the spring has good energy storage and release capabilities, allowing the elastic joint to reach much higher velocities than the rigid counterpart. For low $\sqrt{e_{S L}}$, most of the energy is provided by the motor, i.e. the elastic transmission has only little benefit on the achievable velocity.

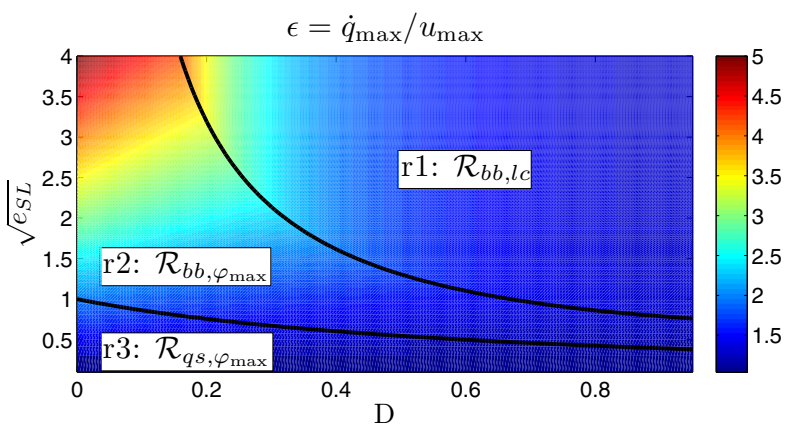

Fig. 3. Maximum link velocity increase for case $r 1$, r2, and r3 depending on the energy ratio $\sqrt{e_{S L}}$ and damping $D$.

In case $\mathrm{r} 1$, the maximum velocity (19) only depends on $D$, as the deflection constraint is not active. If damping is low, the maximum velocity of the limit cycle reaches large values. For $D=0$ the velocity may theoretically become infinite, because there exists no limit cycle [8]. In this situation, the maximum speed is rather bounded by the deflection constraint, since elastic energy is limited. This case is described next.

2) Case r2: In this case, the maximum velocity and reachable set of states are reduced in comparison to the previous one. First, we derive the maximum possible velocity. The according time-optimal trajectory will then help to identify the set of reachable states. Afterwards, the boundary between case $\mathrm{r} 1$ and $\mathrm{r} 2$ is found.

a) Maximum velocity: In order to optimally exploit the elastic energy, it is necessary to fully charge the spring and transform as much potential energy as possible into kinetic link energy. The maximum energetic state the joint can occupy is $\boldsymbol{x}_{c h}:=\left[\begin{array}{l}\dot{q}_{\max } \varphi_{\max }\end{array}\right]^{T}$, see Fig. 4 (upper). As shown in [10], this charged state can be reached by bang-bang control without violating the maximum deflection. Once $\boldsymbol{x}_{c h}$ has been reached, one must apply the maximum motor velocity $u=u_{\max }$, i.e. the link travels in the inertial frame of the motor, to gain maximum speed. Inserting $\boldsymbol{x}_{0}=$ $\boldsymbol{x}_{c h}$ into the system dynamics and setting $u=u_{\max }$, one can derive the maximum velocity

$$
\dot{q}_{\max , \varphi_{\max }}=u_{\max }\left(1+\sqrt{e_{S L}} F_{3}(D)\right),
$$



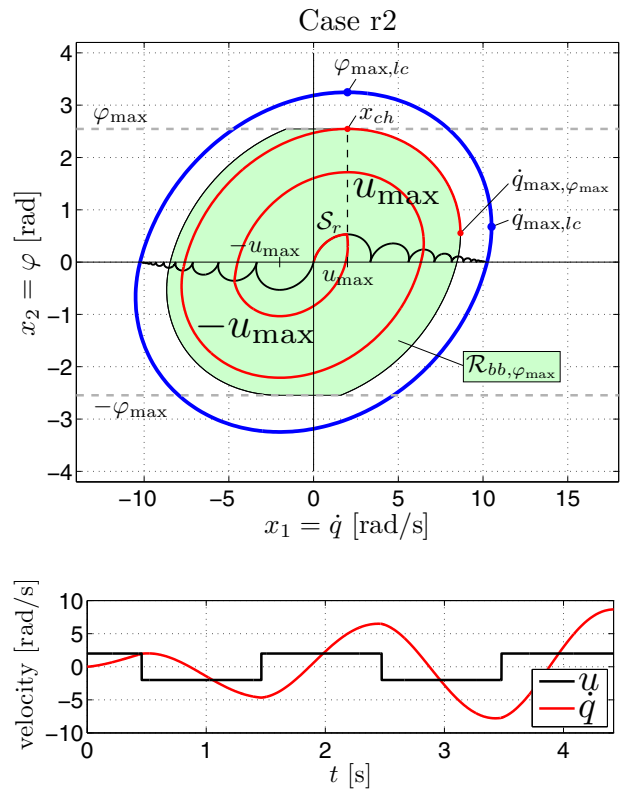

Fig. 4. Reachable states in case the elastic deflection is lower than the maximum deflection of the limit cycle (upper). The blue line indicates the limit cycle, the colored area all reachable states. The red signal depicts the trajectory for reaching the maximum possible velocity starting from the origin. The corresponding timely evolution is shown in the lower figure.

where

$$
F_{3}(D)=e^{-\arctan \left(\frac{\sqrt{1-D^{2}}}{D}\right) \frac{D}{\sqrt{1-D^{2}}}}
$$

The phase plane trajectory for reaching $\dot{q}_{\max , \varphi_{\max }}$ is depicted in Fig. 4 (upper). The corresponding timely evolution of motor and link velocity is shown in the lower figure. The maximum velocity (22) increases linearly with $\sqrt{e_{S L}}$ and decreases exponentially with $F_{3}(D)$. If damping is low, which is the case for most real-world joints, the energy ratio has a much stronger influence on maximum velocity than damping, see Fig. 3.

b) Available energy for velocity maximization: At maximum link velocity the acceleration becomes zero. The elastic deflection at this point can be determined by setting $\ddot{q}=0$ in (7) and solving for $\varphi$, which yields

$$
\varphi\left(\dot{q}_{\max , \varphi_{\max }}\right)=\frac{2 D}{\omega}\left(\dot{q}_{\max , \varphi}-u_{\max }\right) .
$$

This equation shows that the elastic energy can only be utilized to a limited extent if damping is present. Only if $D=0$ we obtain $\varphi\left(\dot{q}_{\max , \varphi_{\max }}\right)=0$ and one can fully transform potential energy to kinetic energy. This fact supports the general design guideline of choosing low damping for robotic systems and realizing it rather via active damping if necessary. Next, we describe the set of reachable states.

c) Reachable set: The trajectory with the largest distance to the origin in the phase plane defines the boundary of the reachable set. The aforementioned trajectory for obtaining the maximum possible link velocity is part of this boundary. Therefore, we start from $\boldsymbol{x}_{c h}$ and apply $u=u_{\max }$ until $\dot{q}_{\max , \varphi_{\max }}$ is hit. Going forward in time, we keep $u=$ $u_{\max }$ until we reach zero deflection, and do not reverse the motor velocity when hitting the switching manifold. Since we are interested in maximizing the distance to the origin and not finding a time-optimal trajectory, this control leads to a larger velocity increase than switching the motor speed when $\mathcal{S}_{r}$ is reached. After hitting $x_{1}=0$ we switch to $u=-u_{\max }$ until the minimum elastic deflection is obtained. Please note that the described boundary is point symmetric w.r.t. the origin. For obtaining the boundary trajectory on the left half of the phase plane, one must start from $\left[\begin{array}{ll}-u_{\max } & -\varphi_{\max }\end{array}\right]^{T}$ and apply the inverse motor input as for the boundary on the right half of the phase plane. Finally, the maximum and minimum elastic deflection define the upper and lower bound of the reachable set.

The boundary of the reachable set in case $\mathrm{r} 2$ can be obtained by bang-bang control, so do all states located within the set. Due to this fact together with the maximum possible velocity being bounded by the maximum elastic deflection, we denote the set of reachable states $\mathcal{R}_{b b, \varphi_{\max }}$. In Fig. 4 this set is represented by the colored area.

d) Boundary between case $r 1$ and $r 2$ : For determining whether the maximum velocity is bounded by a limited cycle (r1) or the maximum elastic deflection (r2), we set $\dot{q}_{\max , \varphi_{\max }}=\dot{q}_{\max , l c}$ and solve for $\sqrt{e_{S L}}$. The boundary

$$
{\sqrt{e_{S L}}}_{r 12}(D)=2 \frac{F_{1}(D)}{\left(1-F_{2}(D)\right) F_{3}(D)}
$$

is a relationship between energy ratio and damping. It is shown as a black solid line between area r1 and r2 in Fig. 3. Below the boundary, the maximum velocity is bounded by the maximum deflection, while above it is bounded by the limit cycle. The remaining area $\mathrm{r} 3$ is described next.

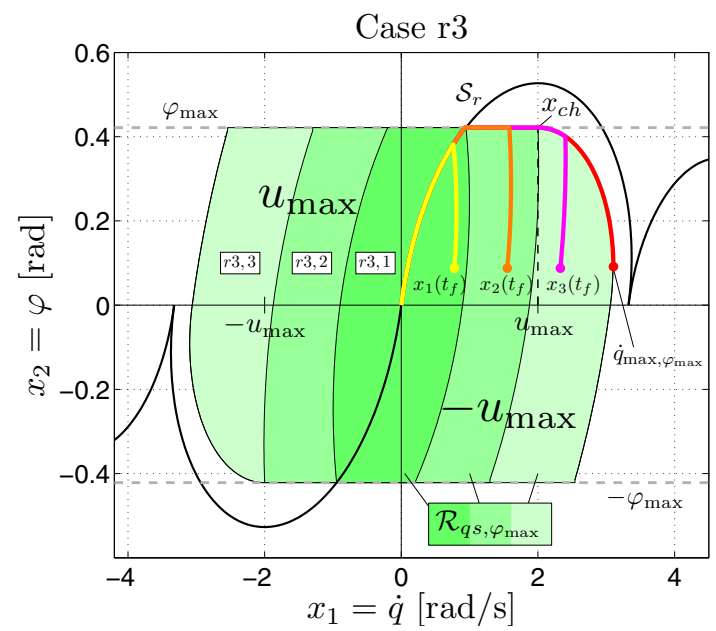

Fig. 5. Reachable states for case r3. States located within the subcase areas can time-optimally be reached as follows: r3,1: bang-bang, r3,2: bangsingular-bang, r3,3: bang-singular-bang-bang. Four trajectories are depicted for illustrating the optimal control in each region. The corresponding timely evolutions of motor and link speed are depicted in Fig. 6.

3) Case r3: In this case the elastic deflection is lowered such that it intersects the switching manifold $\mathcal{S}_{r}$. Bang-bang control can no longer be applied because it would violate the deflection constraint. The maximum deflection of the switching manifold is

$$
\varphi_{\mathcal{S}_{r}, \max }=\frac{u}{\omega} F_{3}(D)
$$

In terms of energy ratio we can rewrite (26) and obtain

$$
{\sqrt{e_{S L} 23}}_{r}(D)=F_{3}(D) \text {. }
$$



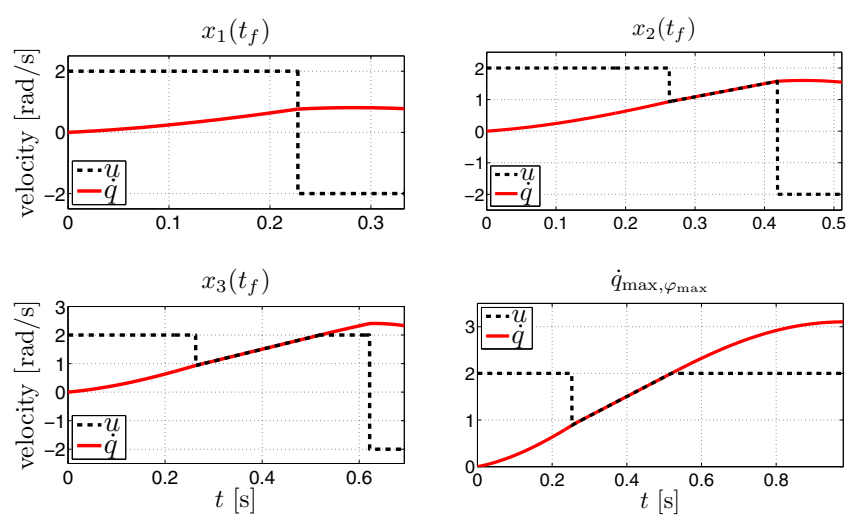

Fig. 6. Exemplary time-optimal trajectories according to Fig. 5.

As for case $r 2$, the maximum velocity is bounded due to the deflection constraint and can therefore be determined with (22). The maximum velocity for case $r 3$ and the boundary $\sqrt{e S L}_{r 23}$ between $\mathrm{r} 2$ and $\mathrm{r} 3$ are depicted in Fig. 3. An example for case $3 r$ is illustrated in Fig. 5. The reachable set of states can be obtained with the same approach as in case r2. However, the maximum velocity cannot be reached by bang-bang control, but requires quasi-singular solution arcs [10]. When hitting the deflection constraint $x_{1}=\varphi_{\max }$, the boundary control $u_{b}=x_{1}$ must be applied, see Sec. II. Maintaining the maximum possible spring deflection means that the maximum elastic torque is used to accelerate the link. Therefore, this is the time-optimal control. The phase-plane trajectory can travel on the constraint until the charged state $\boldsymbol{x}_{c h}=\left[\begin{array}{ll}u_{\max } & \varphi_{\max }\end{array}\right]^{T}$ is reached. For hitting the maximum velocity, one must then apply $u=u_{\max }$, see Fig. 5 .

For hitting states within the reachable set denoted by $\mathcal{R}_{q s, \varphi_{\text {max }}}$, three distinct regions can be identified. In area r3,1 bang-bang control must be applied to reach every state. The outer boundary can be obtained when switching to $u=-u_{\max }$ once the maximum deflection was hit. States located in the second area r3,2 can be hit by bangsingular-bang control, the boundary is defined by the spiral intersecting $\boldsymbol{x}_{c h}$. The third region r 3,3 is bounded by the set of reachable states. Any state belonging to this area can be reached by bang-singular-bang-bang control. In Fig. 5, four trajectories are illustrated to show the time-optimal solution for each area. The corresponding timely evolutions of states are depicted in Fig. 6.

\section{B. Braking to equilibrium}

Having found the reachable states and how to obtain them time-optimally starting from equilibrium, we now consider the inverse problem of hitting a resting position as fast as possible. As mentioned in Sec. II, the initial state before braking may be hit either by a trajectory found for the previous problem or caused by an external collision. For braking we can identify two cases:

b1) all states can be stopped by bang-bang control

b2) quasi-singular solutions may occur

In contrast to the reaching problem, there exists no limit cycle, cases b1 and b2 share the same goal $\boldsymbol{x}\left(t_{f}\right)=\left[\begin{array}{ll}0 & 0\end{array}\right]^{T}$. The switching manifold referred to as $\mathcal{S}_{b}$ can be obtained in an analogous manner to problem I. We get the first curves of
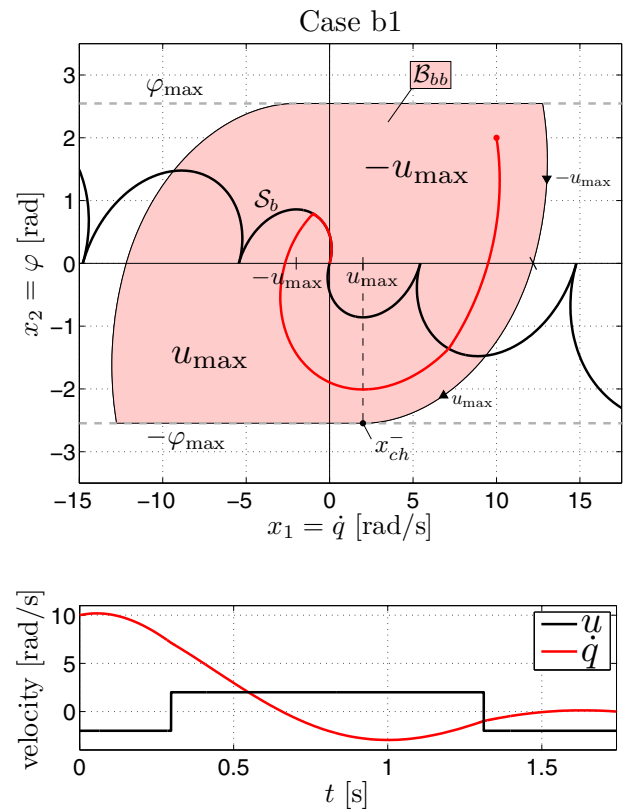

Fig. 7. The colored area in the upper figure represents all brakable states (case b1). The red solid line is an exemplary trajectory for reaching the origin in minimum time. The corresponding timely evolution of motor and link speed is depicted in the lower figure.

the manifold when starting from $\left[\begin{array}{ll}0 & 0\end{array}\right]^{T}$ and going backwards in time with $u= \pm u_{\max }$ for $t=t_{s}$. Above the switching manifold, one must apply $u=-u_{\max }$, below $\mathcal{S}_{b} u=u_{\max }$ applies to hit the steady state in minimum time, see Fig. 7 (upper).

Next, we find the outer boundary of the brakable set. The approach is the same for cases b1 and b2.

1) Brakable set: In the aforementioned problem of reaching desired states, one important point on the boundary was $\boldsymbol{x}_{c h}=\left[\begin{array}{ll}u_{\max } & \varphi_{\max }\end{array}\right]^{T}$. This maximum energetic state must be hit asymptotically in order to reach the maximum velocity, which lies on the boundary of the reachable set. Also for this problem, the trajectory which has the largest distance to the origin and therefore defines the boundary of $\mathcal{B}$ reaches the marginal deflection asymptotically. Since the system trajectory is moving clockwise in the phase plane, this state is denoted $\boldsymbol{x}_{c h}^{-}=\left[\begin{array}{ll}u_{\max } & -\varphi_{\max }\end{array}\right]^{T}$. The boundary can now be obtained as follows. In a reversed time evolution starting from $\boldsymbol{x}_{c h}^{-}$, we apply $u=u_{\max }$ until we reach zero deflection, see Fig. 7 (upper). Then, we switch to $u=-u_{\max }$ until the maximum deflection is hit. The boundary is point symmetric w.r.t. the origin, the inverse procedure gives the boundary on the left hand side of the phase plane. The upper and lower boundary of the brakable set are finally defined by the maximum and minimum elastic deflection, respectively.

2) Case b1: Analogous to problem I, bang-bang control is possible for all brakable states if the first curve of the switching manifold can be hit without violating the motor velocity or deflection constraint. This is the case if the maximum elastic deflection is larger than the maximum deflection of the first switching curve. The boundary between case b1 and b2 in terms of energy and damping ratio is

$$
{\sqrt{e_{S L}}}_{b 12}(D)=e^{\frac{D}{\sqrt{1-D^{2}}}\left(\pi-\arctan \left(\frac{\sqrt{1-D^{2}}}{D}\right)\right)} .
$$


An example where all states within the brakable set can be stopped by bang-bang control is depicted in Fig. 7 (upper). The colored area represents the brakable set denoted $\mathcal{R}_{b b}$.

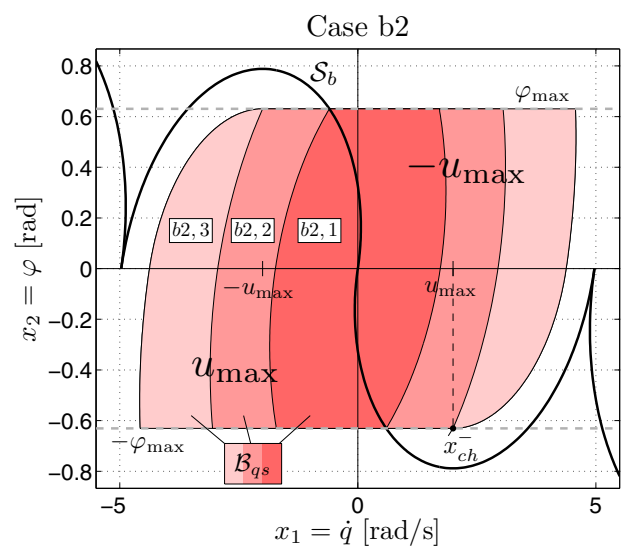

Fig. 8. Brakable states for case b2. States located within the subcase areas can time-optimally be stopped as follows: b2,1: bang-bang, b2,2: bangsingular-bang, b2,3: bang-bang-singular-bang.

3) Case b2: If the elastic deflection intersects the first switching curve of $\mathcal{S}_{b}$, we may obtain quasi-singular solution pieces because bang-bang control can violate the constraint. An example for case b2 is illustrated in Fig. 8. We can identify three subcases (b2,1, b2,2, and b2,3). The brakable set $\mathcal{B}_{q s}$ and the according time-optimal control can be derived similar to case $\mathrm{r} 3$.

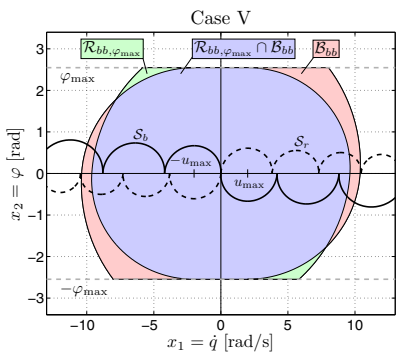

(a) $\sqrt{e_{S L}}=4, D=0.03$

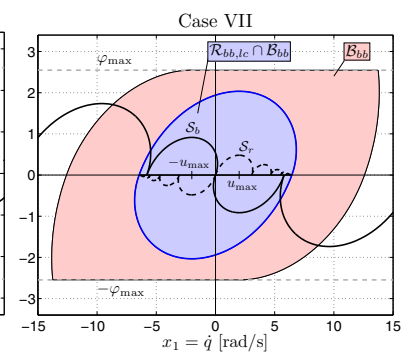

(b) $\sqrt{e_{S L}}=4, D=0.2$
Fig. 9. Two exemplary combinations of reachable and brakable sets.

\section{Reachable vs. brakable states}

In Fig. 9 a) we show an example where both the reachable and brakable set are depicted for a given joint design. Bang-bang control is possible for both sets. The blue area represents states which are reachable and brakable, however, there also exist states that can be reached but not be returned to the equilibrium (green area) and vice versa (red area). If damping is being increased for the considered joint as shown in Fig. 9 b), the reachable set becomes a subset of the brakable set $(\mathcal{R} \subset \mathcal{B})$. The condition for this case is as follows. The boundary of the brakable set always hits $\left[u_{\max }-\varphi_{\max }\right]^{T}$ in the fourth quadrant, as described the previous section. The reachable set is a subset of the brakable set if the boundary of the reachable set intersects the minimum elastic deflection at $\dot{q}\left(-\varphi_{\max }\right) \geq u_{\max }$. The analytic formulation of the boundary in terms of $\sqrt{e_{S L}}$ and $D$ is omitted for brevity.

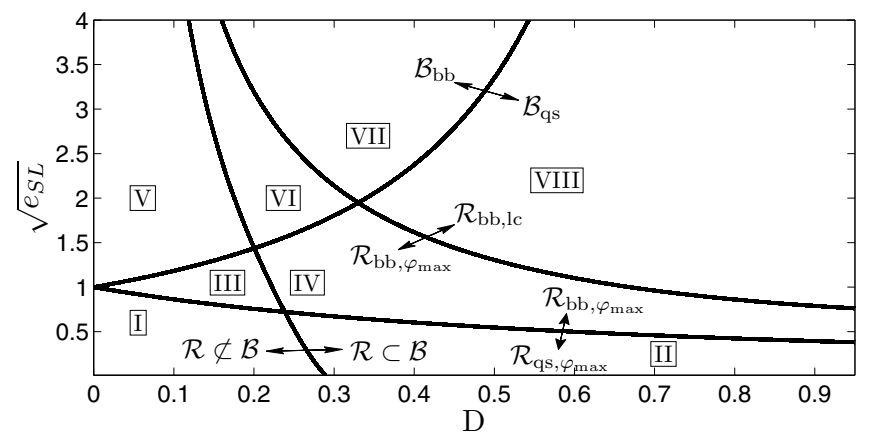

Fig. 10. Cases for the reachable and brakable set of states, depending on energy ratio and damping.

For determining the influence of system parameters on the overall performance, we summarize our results in Fig. 10. Boundaries (25), (27) are shown for all reaching cases, boundary (28) for the braking cases, and finally the boundary whether $\mathcal{R} \subset \mathcal{B}$ holds. In total, we can identify eight distinct cases, depending on $\sqrt{e_{S L}}$ and $D$. From Fig. 10, one can derive which concrete design parameters should be selected in order to achieve a desired behavior. Regions I and II are less desirable because quasi-singular solutions pieces may exist for both reaching and braking. Bang-bang control can be applied for reaching desired states if the joint design is located in region III-VIII, and for braking if region V-VII is selected. Thus, reaching desired states and braking to the equilibrium is always possible with bang-bang excitation if the joint design is located in region V, VI, or VII. For maximum velocity increase, $\mathrm{V}$ is the most desirable region because the joint has good energy storage and release capabilities and low damping, c.f. Fig. 3 and Fig. 9 a).

\section{NEAR TIME-OPTIMAL BRAKING FOR ELASTIC $n$-DOF MANIPULATORS}

In this section, we apply the theory developed in the previous section to $n$-DOF manipulators. We seek to stop an elastic robot as fast as possible. Clearly, braking every joint leads to a velocity decrease of the entire robot. As braking is a very local behavior, we may assume $\boldsymbol{g}(\boldsymbol{q})=$ const. along the braking trajectory, which can then be easily compensated. Furthermore, a negligible centrifugal and Coriolis torque is considered, i.e. $C(\boldsymbol{q}, \dot{\boldsymbol{q}})=\mathbf{0}, \boldsymbol{\tau}_{\text {ext }}=\mathbf{0}$. The reduced dynamics are therefore

$$
\begin{aligned}
\boldsymbol{\theta} & =\int \dot{\boldsymbol{\theta}} d t+\boldsymbol{\theta}_{0} \\
M(\boldsymbol{q}) \ddot{\boldsymbol{q}} & =K_{J}(\boldsymbol{\theta}-\boldsymbol{q}),
\end{aligned}
$$

with $\boldsymbol{\theta}_{0} \in \mathbb{R}^{n}$ being the initial motor position at the time braking is initialized.

Generally, (29)-(30) cannot be solved analytically due to the highly nonlinear inertial coupling via $M(\boldsymbol{q})$. Solving the problem numerically is no option, as this will not be possible in real-time. Therefore, we propose a solution to the problem, which is slightly suboptimal, however able to compute the braking trajectory instantaneously. 

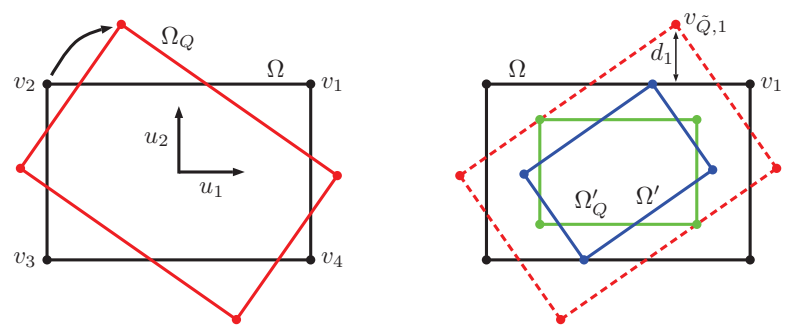

Fig. 11. Original and transformed control region (left), and obtaining a symmetric control region in decoupled space via scaling (right).

\section{A. Dynamics decoupling}

To simplify the optimal control problem, we decouple the dynamics by making use of the generalized eigenvalue problem. Given the symmetric positive definite mass matrix $M$ and the positive definite stiffness matrix $K$, an orthonormal maxtrix $Q \in \mathbb{R}^{n \times n}$ can be found such that

$$
M Q=K Q M_{Q}
$$

where $M_{Q}$ is a diagonal matrix. Using the new decoupled coordinates $\boldsymbol{\theta}_{Q}=Q^{-1} \boldsymbol{\theta}$ and $\boldsymbol{q}_{Q}=Q^{-1} \boldsymbol{q}$ we obtain $n$ independent SISO mass-spring systems

$$
M_{Q} \ddot{\boldsymbol{q}}_{Q}=\boldsymbol{\theta}_{Q}-\boldsymbol{q}_{Q}
$$

Important to notice is that also the control region has to be transformed into the decoupled space. The control region in the original space is defined as the hypercube

$$
\Omega:=\left[u_{1, \min }, u_{1, \max }\right] \times \cdots \times\left[u_{n, \min }, u_{n, \max }\right],
$$

associated to the vertex vector $\boldsymbol{v}_{i}=\left[v_{i}(1), \ldots, v_{i}(n)\right]^{\mathrm{T}}, i=$ $1 \ldots 2^{n}$. The transformed control region is defined as

$$
\Omega_{Q}:=Q^{-1}(\Omega),
$$

meaning every line segment of $\Omega$ is being transformed. The transformation is linear, for $\operatorname{det} Q^{-1}=1$ the hypercube is being rotated and for $\operatorname{det} Q^{-1}=-1$ it is being reflected. Figure 11 depicts an example for $n=2$ (e.g. a double pendulum) with $\operatorname{det} Q^{-1}=1$.

\section{B. Control region decoupling}

Note that after transformation into decoupled space the maximum/minimum values of each control input are no longer independent from each other, see Fig. 11 (left). Thus, the decoupling property does not apply to the admissible control region. Surely, one could formulate an optimal control problem including constraints on the control inputs. However, we want to maintain the decoupling property for solving the problem analytically and therefore search for the largest hypercube $\Omega_{Q}^{\prime}$ in the transformed control region that lies completely in the original bounds in retransformed space $\left(\Omega^{\prime}\right)$. This ensures independent maxima/minima control inputs for each subsystem, c.f. Fig. 11 (right). The maximum control input in decoupled space is obtained via Algorithm 1 , where $k$ is the overall scaling factor.

The bounds of the new control region are found to be

$$
\frac{\min \left\{\boldsymbol{u}_{\max }(1), \ldots, \boldsymbol{u}_{\max }(n)\right\}}{\left\|\boldsymbol{u}_{\max }\right\|_{2}} \boldsymbol{u}_{\max } \leq \boldsymbol{u}_{Q, \max }^{\prime} \leq \boldsymbol{u}_{\max }
$$

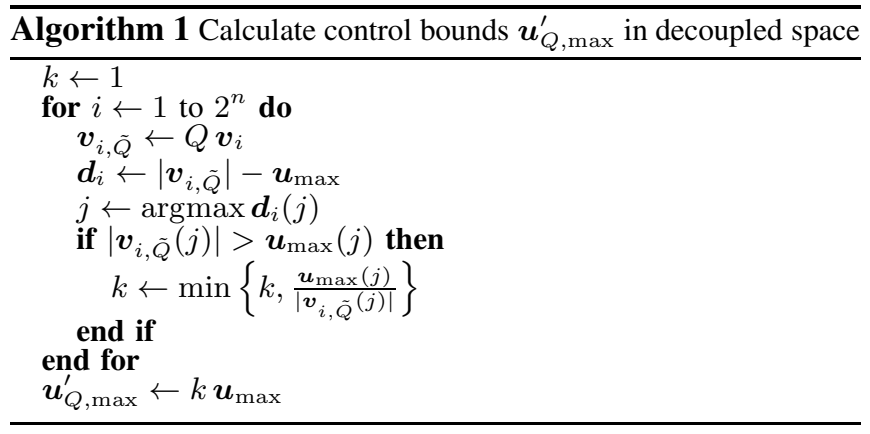

Having obtained $n$ SISO subsystems with constant input constraints, the optimal control problem can be solved. While the proposed approach can be implemented in real-time, it is suboptimal in terms of braking time due to following reasons:

- Instead of global optimization, a local optimization for each joint is carried out.

- In order to solve the optimal control problem for every joint separately, the control region needs to be reduced to maintain decoupling.

- Gravity and Coriolis torques have been neglected, as we assume their effect to be quasi-stationary/negligible and therefore they are compensated.

In the following, we present the full controller structure and apply it to a 7-DOF robot.

\section{Experimental verification}

For demonstrating the controller performance, we conducted an experiment with a KUKA/DLR LWR4. We use active joint impedance control for emulating significant joint elasticity similar to robots with intrinsic joint elasticity. For the experiment, we select $K_{J, i}=200 \mathrm{Nm} / \mathrm{rad} \forall i \in$ $\{1, \ldots, 7\}$ stiffness. In order to analyze the performance of the controller only, we select $D_{i}=0, \forall i \in\{1, \ldots, 7\}$. If damping were present, the robot would even stop without the braking controller being active. In the previous section we argued in favor of joint designs where both accelerating and braking are possible by applying bang-bang control. For now, we assume that such a design was chosen and therefore do not need to take deflection constraints into account.

The real-time capable controller structure is depicted in Fig. 12. The robot dynamics are linearized in every time step. First, the dynamics are being decoupled according to (31). Then, the control region is being decoupled (algorithm 1). Now, we have seven SISO systems and apply the braking controller independently to every joint. Please note that there are no gains or parameters to influence the controller behavior since it is optimal (on joint level). Having found the time-optimal control input $\boldsymbol{u}_{Q}^{*}$ we retransform the input into the original space, integrate and forward it to the joint impedance controller, which has a position interface.

Some experimental results are depicted in Fig. 13. This experiment can also be found in the attached video. In the experiment, we apply an external force to the undamped robot while the braking controller is deactivated. This causes significant oscillations in every joint. When enabling the braking controller, the joint velocities and deflections quickly $(\approx 400 \mathrm{~ms})$ decay to zero. Although the controller is of bang- 


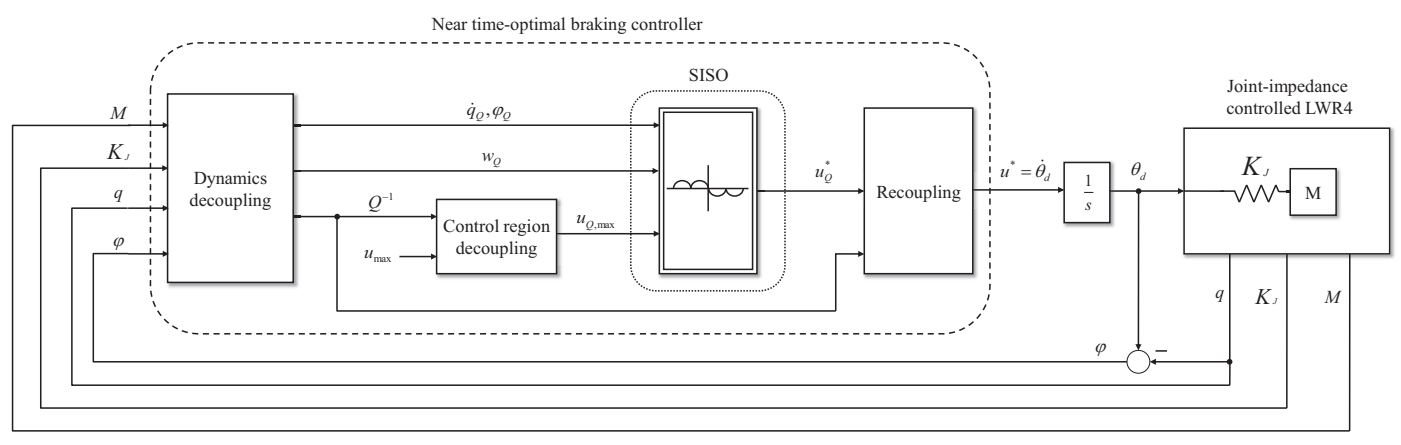

Fig. 12. Controller structure for braking a joint-impedance controlled KUKA/DLR LWR4 in near-minimum time.

bang type on velocity level, we obtain feasible desired motor positions, see Fig. 13.
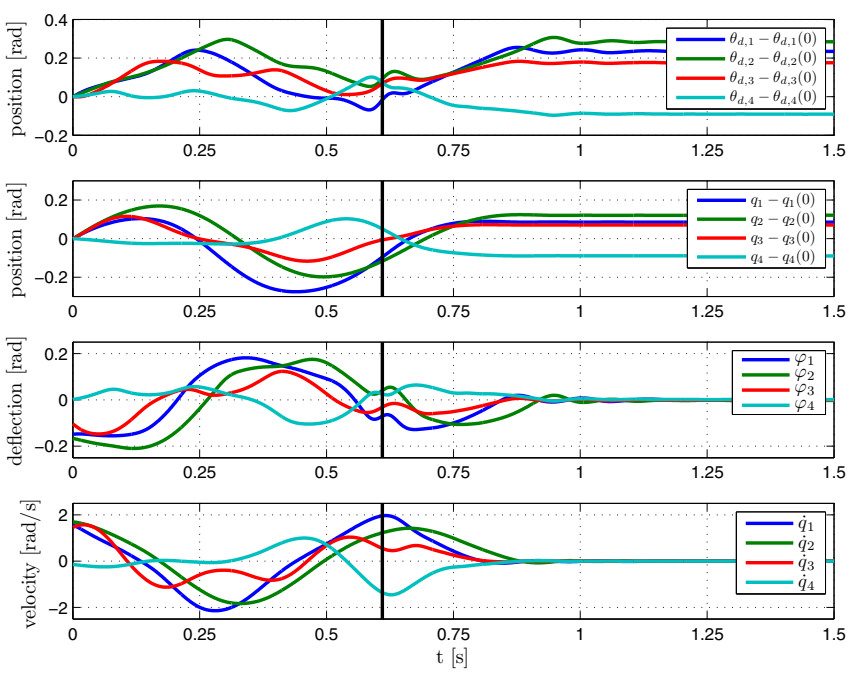

Fig. 13. Recorded signals from the experiment with a KUKA/DLR LWR4. The change of the desired motor position $\boldsymbol{\theta}_{d}-\boldsymbol{\theta}_{d}(0)$ is depicted in the top figure, the change of link position $\boldsymbol{q}-\boldsymbol{q}(0)$ in the second, the elastic deflection $\varphi$ proportional to $\tau_{J}$ in the third, and the joint velocities $\dot{\boldsymbol{q}}$ in the bottom figure. The black vertical line indicates when the braking controller is activated. We only show the signals of the first four joints, since the collision almost had no influence on the other joints. However, the behavior of the entire robot can also be seen in the attached video.

\section{CONCLUSION}

In this paper, the problems of reaching desired states of visco-elastic joints from equilibrium and the according inverse problem of which states can be brought to a resting state in a time-optimal manner were solved. For both problems we gave basic insight into the influence of elastic energy, motor velocity, joint stiffness, and damping. The presented results provide clear information on how to select system parameters such that both, good energy storage and release (dissipation) capabilities can be achieved. The developed 1-DOF theory was extended to nonlinear $n$ DOF elastic manipulators with having the goal of real-time capability in mind. By decoupling the dynamics and control region, a controller was derived which is able to brake an elastic manipulator in near-minimum time. The controller is verified experimentally with a KUKA/DLR LWR4 in joint impedance control. Our future work will consider the stability analysis of the proposed controller and deal with nonlinear and/or variable stiffness characteristics (with deflection constraints), e.g. the DLR Hand Arm System.

\section{ACKNOWLEDGMENT}

This work has been partially funded by the European Commission's Seventh Framework Programme as part of the project SAPHARI under grant no. 287513.

\section{REFERENCES}

[1] A. Bicchi and G. Tonietti, "Fast and soft arm tactics: Dealing with the safety-performance trade-off in robot arms design and control," IEEE Robotics and Automation Mag., vol. 11, pp. 22-33, 2004.

[2] B. Vanderborght, B. Verrelst, R. Van Ham, M. Van Damme, D. Lefeber, B. M. Y. Duran, and P. Beyl, "Exploiting natural dynamics to reduce energy consumption by controlling the compliance of soft actuators," The International Journal of Robotics Research, vol. 25 , no. 4, pp. 343-358, 2006.

[3] N. Tsagarakis, M. Laffranchi, B. Vanderborght, and D. G. Caldwell, "A compact soft actuator unit for small scale human friendly robots," in IEEE International Conference on Robotics and Automation, (ICRA 2009), Kobe Japan, 2011, pp. 4356-4362.

[4] H. Iwata and S. Sugano, "Design of human symbiotic robot TWENDYONE," IEEE Int. Conf. on Robotics and Automation (ICRA 2009), Kobe, Japan, pp. 580-586, 2009.

[5] M. Grebenstein, A. Albu-Schaffer, T. Bahls, M. Chalon, O. Eiberger, W. Friedl, R. Gruber, S. Haddadin, U. Hagn, R. Haslinger et al., "The dlr hand arm system," in Robotics and Automation (ICRA), 2011 IEEE International Conference on. IEEE, 2011, pp. 3175-3182.

[6] S. Haddadin, F. Huber, and A. Albu-Schaffer, "Optimal control for exploiting the natural dynamics of variable stiffness robots," in Robotics and Automation (ICRA), 2012 IEEE International Conference on. IEEE, 2012, pp. 3347-3354.

[7] S. Haddadin, T. Laue, U. Frese, S. Wolf, A. Albu-Schäffer, and G. Hirzinger, "Kick it with elasticity: Safety and performance in human-robot soccer," Robotics and Autonomous Systems, vol. 57, no. 8, pp. 761-775, 2009.

[8] S. Haddadin, M. Weis, S. Wolf, and A. Albu-Schäffer, "Optimal control for maximizing link velocity of robotic variable stiffness joints," in IFAC World Congress, 2011.

[9] M. C. Ozparpucu and S. Haddadin, "Optimal control for maximizing link velocity of visco-elastic joints," in IEEE/RSJ International Conference on Intelligent Robots and Systems (IROS), 2013, pp. 30353042.

[10] S. Haddadin, K. Krieger, N. Mansfeld, and A. Albu-Schaffer, "On impact decoupling properties of elastic robots and time optimal velocity maximization on joint level," in IEEE/RSJ International Conference on Intelligent Robots and Systems (IROS), 2012. IEEE, 2012, pp. 5089-5096.

[11] S. Haddadin, A. Albu-Schäffer, O. Eiberger, and G. Hirzinger, "New insights concerning intrinsic joint elasticity for safety," in IEEE/RSJ Int. Conf. on Intelligent Robots and Systems (IROS2010), Taipeh, Taiwan, 2010, pp. 2181-2187.

[12] R. F. Hartl, S. P. Sethi, and R. G. Vickson, "A survey of the maximum principles for optimal control problems with state constraints," SIAM review, vol. 37, no. 2, pp. 181-218, 1995

[13] D. Kirk, Optimal control theory. Prentice-Hall, 1970. 